\title{
A systematic review
}

-Agamemnon Pericleous ${ }^{1}$,Timothy Davis ${ }^{2}$,Mat Daniel ${ }^{3}$,Johanna Barry ${ }^{4}$

-1 Department of Otolaryngology, Queen's Medical Centre, Nottingham University Hospitals NHS Trust

- 2 University of Nottingham, Medical School

-3 Department of Otolaryngology, Queen's Medical Centre, Nottingham University Hospitals NHS Trust; MRC Institute of Hearing Research, University of

Nottingham

.4 MRC Institute of Hearing Research, University of Nottingham.

\section{Introduction}

Whether OME affects a child's educational achievement, behaviour or speech and language development is unclear. Various studies aimed to assess the impact of OME in various aspects of development with conflincting results.

\section{The aim}

Aim was to undertake a systematic review to analyse the findings of published studies.

\section{Methods}

Embase and MEDLINE search , including studies involving otherwise-healthy and typically-developing children. The screening process was carried out as per PRISMA protocol. Screening was carried out by two independent assessors. Information such as study design, whether unilateral/bilateral OME was assessed, presence of intervention, age of participants, domain assessed and effect was extracted.

\section{Discussion}

Large methodological variation between studies makes interpretation difficult.

5 studies showed a significant effect on developmental outcomes for children with bilateral OME.

Only 1 study was composed entirely of children with bilateral hearing loss lasting 3 months or longer with hearing of $25 \mathrm{~dB}$ or worse in the better hearing ear and this study showed a significant impact.

The other 9 studies either included children with better hearing or it was not specified.

\section{Conclusions}

10 studies involving the effect of bilateral OME on the developmental outcomes of children were analysed. Of these, a significant effect was found in $\mathbf{5}$ studies

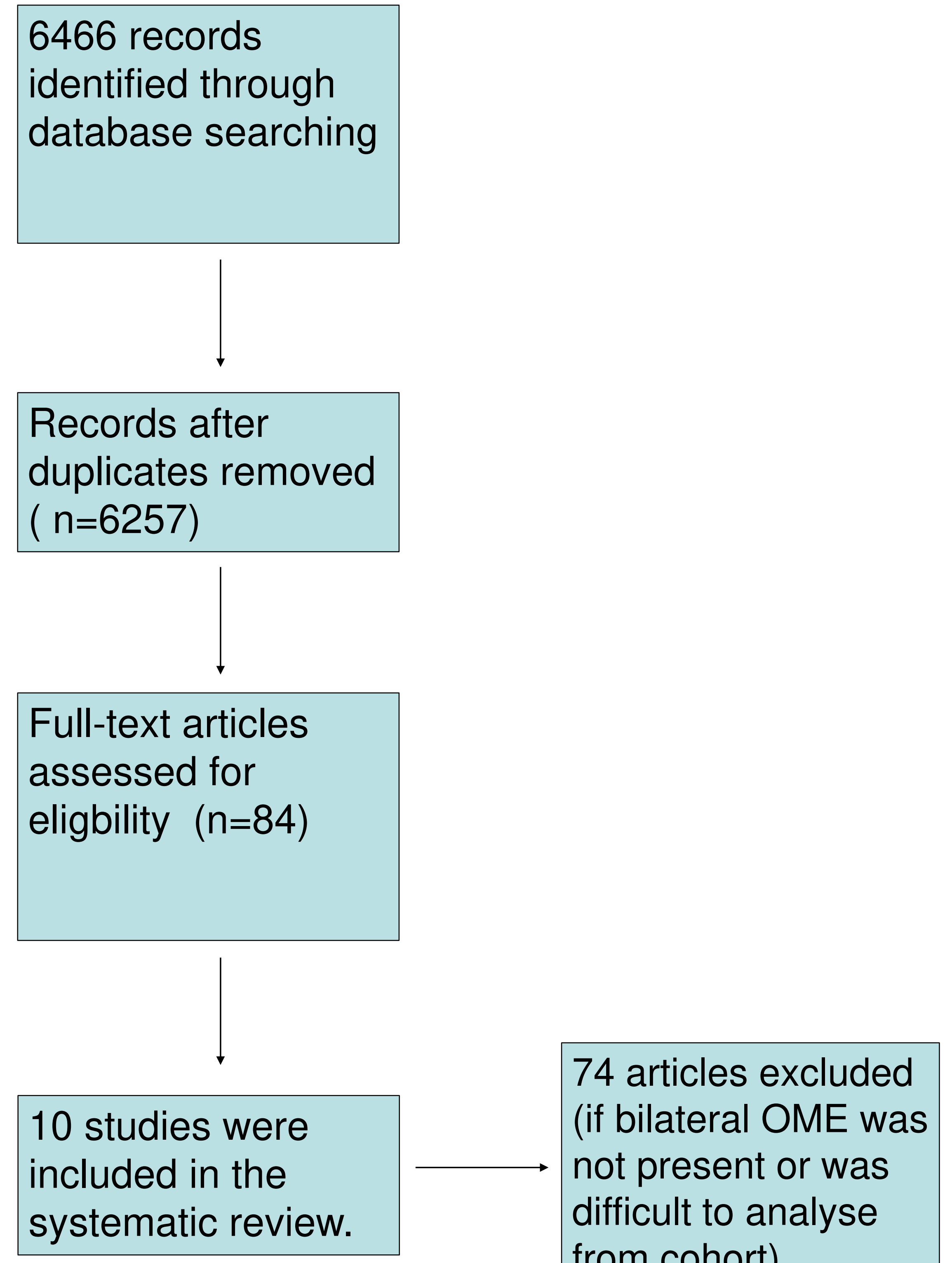

systematic review.

The table below illustrates the studies that were included following assessment for eligibility.

\begin{tabular}{|c|c|c|c|c|}
\hline AUTHORS & JOURNAL & NICE CRITERIA & DOMAIN & EFFECT \\
\hline McCormick DP et al & $\begin{array}{l}\text { Ambulatory pediatrics } 1 \text { (2) (pp 87- } \\
90), 2001\end{array}$ & UNKNOWN & language & significant effect \\
\hline Grievink E.H. et al & $\begin{array}{l}\text { Journal of Speech and Hearing } \\
\text { Research. } 36 \text { (5) (pp 1004-1012), } \\
1993 .\end{array}$ & MIXED & language & no effect \\
\hline Gravel JS & $\begin{array}{l}\text { Journal of Speech and Hearing } \\
\text { Research. } 35 \text { (3) (pp 588-595), } \\
1992 .\end{array}$ & UNKNOWN & language & Significant effect \\
\hline Wallace IF et al & $\begin{array}{l}\text { Laryngoscope. } 98 \text { (1) (pp 64-70), } \\
1988 .\end{array}$ & UNKNOWN & Language, cognition & Significant effect \\
\hline Johnson DL et al & $\begin{array}{l}\text { Journal of Communication } \\
\text { Disorders. 41(1):20-32, } 2008 \text { Jan- } \\
\text { Feb }\end{array}$ & UNKNOWN & language & No significant effect \\
\hline Paradise JL et al & $\begin{array}{l}\text { Pediatric Infectious Disease } \\
\text { Journal. 22(4):309-14, } 2003\end{array}$ & MIXED & Language, cognition & No significant effect \\
\hline Asbjiordsen A et al & $\begin{array}{l}\text { Developmental Medicine \& Child } \\
\text { Neurology. } 42(7): 481-6,2000\end{array}$ & UNKNOWN & attention & Significant effect \\
\hline Augustsson I et al & $\begin{array}{l}\text { International Journal of Pediatric } \\
\text { Otorhinolaryngology. } 57 \text { (1) (pp 31- } \\
\text { 40), 2001. }\end{array}$ & UNKNOWN & Academic & No significant effect \\
\hline Asbjornsen $\mathrm{A}$ et al & $\begin{array}{l}\text { Child Neuropsychology. } 11 \text { (2) (pp } \\
\text { 121-133), } 2005 .\end{array}$ & UNKNOWN & Attention & No significant effect \\
\hline Maw $\mathrm{R}$ et al & Lancet. 353(9157):960-3, 1999 & YES & Language & Significant effect \\
\hline
\end{tabular}

Flow diagram illustrating screening process of articles as per PRISMA protocol (Figure 1) 